\title{
Clinical Global Impression - Corrections (CGI-C) - deutsche Übersetzung
}

\author{
Carola Billen ${ }^{1}$ Michel A. Schulte-Ostermann ${ }^{2} \cdot$ Christian Huchzermeier $^{2}$ \\ Eingegangen: 21. Dezember 2019 / Angenommen: 16. März 2020 / Online publiziert: 29. April 2020 \\ (c) Der/die Autor(en) 2020
}

\section{Zusammenfassung}

In mehreren Studien wurden hohe Prävalenzen psychiatrischer Störungen in den Justizvollzugsanstalten sowie der Mangel an adäquaten Behandlungsoptionen festgestellt. Jedoch blieb bisher unklar, inwieweit diese Erkrankungen die Betroffenen einschränken. Um den Schweregrad der Erkrankungen erfassen zu können, wurde das allgemeinpsychiatrisch viel genutzte Instrument „Clinical Global Impression (CGI)“ an die Besonderheiten des Vollzugs (CGI-Corrections, CGI-C) angepasst, da dieses Setting die psychiatrische Versorgung und auch Diagnostik vor große Herausforderungen stellt. Gemäß den Leitlinien der WHO wurden die englische Version des Instruments übersetzt sowie 21 Fallvignetten und anschließend die „Interrater“-Reliabilität mit einer Stichprobe von 20 Personen überprüft. Die Resultate zeigen eine hohe Beurteilerübereinstimmung (Gwet's $\mathrm{AC}_{2}$ 0,82, 96\%-KI 0,74-0,91, $p<0,001$ ) und nur vereinzelte Anmerkungen der Rater/Raterinnen (20 bei insgesamt 420 Ratings), sodass davon auszugehen ist, dass der CGI-C ein schnelles und effizientes Instrument zur Erfassung der Schwere einer psychischen Erkrankung im Vollzugssetting ist. Ziel des Beitrags ist es, die übersetzte Version des Instruments vorzustellen und zur Verfügung zu stellen. Für die Zukunft sind weitere Untersuchungen gefordert, die sich mit den Testgütekriterien in verschiedenen Populationen (z. B. Frauen, Jugendliche), anderen Ratern/Raterinnen (z. B. Sozialarbeiter, Justizvollzugsbeamte) sowie der Retest-Reliabilität beschäftigen.

Schlüsselwörter Rating Skala $\cdot$ Diagnostik $\cdot$ Justizvollzug $\cdot$ Psychiatrische Erkrankung $\cdot$ Schweregrad

Die Autoren C. Billen und M.A. Schulte-Ostermann haben zu gleichen Teilen zum Manuskript beigetragen.

Zusatzmaterial online Zusätzliche Informationen sind in der Online-Version dieses Artikels (https://doi.org/10.1007/s11757020-00599-9) enthalten.

Carola Billen, M. Sc. Forensic Psychology

Carola.Billen@1vr.de

Michel A. Schulte-Ostermann, M. Sc. Forensic Psychology Michel.SchulteOstermann@uksh.de
Prof. Dr. Christian Huchzermeier Christian.Huchzermeier@uksh.de

1 Abteilung für Forensische Psychiatrie und Psychotherapie II, LVR-Klinik Köln, Wilhelm-Griesinger-Straße 23, 51109 Köln, NordrheinWestfalen, Deutschland

2 Institut für Sexualmedizin und Forensische Psychiatrie und Psychotherapie (ISFP), Universitätsklinikum Schleswig-Holstein - Campus Kiel, Niemansweg 147, 24105 Kiel, Schleswig-Holstein, Deutschland 


\title{
Clinical global impression-Corrections (CGI-C)—German version
}

\begin{abstract}
Numerous studies have shown a high prevalence of mental illness within correctional settings and a lack of adequate treatment options; however, there has not been research on or assessment of the severity of the psychiatric symptoms. In order to provide an instrument that measures the severity of mental illness, the often used instrument clinical global impression (CGI) has been adapted to the unique challenges of prison settings (CGI corrections, CGI-C). The authors translated the English version of the instrument into German according to the WHO guidelines. A total of 20 psychologists and psychiatrists rated 21 case vignettes to assess interrater reliability. The results showed a high interrater reliability (Gwet's $\mathrm{AC}_{2}$ 0.82, 96\% CI $0.74-0.91, p<0.001$ ) and only a few comments by the raters regarding the items of the instrument (20 out of 420 ratings). Hence, it must be assumed that the CGI-C is a quick and effective instrument to assess the severity of mental illness in correctional settings. The aim of this technical report is to present the translated version of the CGI-C to a specialized audience. Additionally, further studies are necessary to validate the instrument in different populations (e.g. female or youth offenders), with multidisciplinary raters (e.g. social workers, prison officers) and look at the re-test reliability of the CGI-C.
\end{abstract}

Keywords Rating scale $\cdot$ Assessment $\cdot$ Correctional settings $\cdot$ Mental illness $\cdot$ Severity

\section{Einleitung}

Eine Vielzahl von Studien belegt die hohe Prävalenz psychischer Erkrankungen in Justizvollzugsanstalten (Fazel et al. 2006; Fazel und Danesh 2002; Fazel et al. 2016; Fazel und Seewald 2012; Goff et al. 2007; Huchzermeier et al. 2016; Köhler et al. 2009; Schulte-Ostermann und Huchzermeier 2019; von Schönfeld et al. 2006). Diese Studien liefern zuverlässige Daten über die Prävalenz psychiatrischer Diagnosen gemäß ICD-10 in Gefängnissen, beinhalten aber keine Aussagen über den Schweregrad dieser Erkrankungen. Entsprechend den Erkenntnissen bei allgemeinpsychiatrischen Populationen ist auch bei psychiatrisch erkrankten Gefängnisinsassen von erheblichen Funktionseinschränkungen auszugehen. Um diese Funktionseinbußen zu erfassen, musste bisher auf Instrumente zurückgegriffen werden, die im zivilpsychiatrischen Kontext entwickelt wurden und sich dort bewährt haben, wie z.B. das Global Assessment of Functioning (GAF) oder der Clinical Global Impression (CGI; Janssen et al. 1998; Kamath et al. 2013; MacKain und Baucom 2008; Nelson et al. 2004; Rittmannsberger et al. 2014; Trestman et al. 2007). Der CGI kann zudem für alle Diagnose- und Altersgruppen verwendet werden (Scalarum Collegium Internationale Psychiatriae 2015).

Einschränkungen, die durch die Inhaftierung und deren Besonderheiten entstehen (z. B. fehlender familiärer Kontakt, Probleme, Freundschaften zu halten, Arbeitsplatzverlust) und somit einen Einfluss auf die Beurteilung der Items haben können, werden in diesen Instrumenten jedoch nicht berücksichtigt. Daher sind diese Instrumente für eine valide Einschätzung des Schweregrads der Beeinträchtigung durch psychiatrische Erkrankungen in dem besonderen Lebensumfeld eines Gefängnisses zumeist ungeeignet. Sie können entweder zu einer Unter- oder Überschätzung der Kriterien führen, da der Haftkontext nur ungenügend berücksichtigt wird. Im angelsächsischen Raum veranlasste dieser Mangel Jones et al. (2019) dazu, eine Adaptation der Skala, die die Schwere der psychischen Erkrankung misst, des CGI für das Setting des Justizvollzugs zu entwickeln, den CGICorrections (CGI-C), welche im Jahre 2018 veröffentlicht wurde.

Auch im deutschsprachigen Raum ist für die psychiatrische oder psychotherapeutische Arbeit bisher kein analoges Instrument zur Beurteilung des Schweregrads psychischer Krankheiten etabliert, das die besonderen Rahmenbedingungen von Justizvollzugsanstalten beachtet. Aufgrund erster positiver Ergebnisse in den Pilotuntersuchungen der kanadischen Autoren wurde der CGI-C in enger Kooperation mit Simpson und Jones Ende 2018 für die Nutzung im deutschsprachigen Raum übersetzt.

Ziel dieses Artikels ist es, den Übersetzungsprozess zu beschreiben, die deutschsprachige Version vorzustellen sowie erste Resultate der Interrater-Reliabilität darzustellen, welche anhand von Fallvignetten ermittelt wurden.

\section{Unterschiede zur CGI-Skala}

Die klinische Beurteilung des CGI orientiert sich an der zugehörigen Patientengruppe und erfasst sowohl die beobachteten als auch die berichteten Symptome des Patienten. In Erweiterung dieser Bewertungsgrundlagen beinhaltet der CGI-C ausdrücklich fremdanamnestische Angaben von Dritten (z.B. Bedienstete des Vollzugdiensts), um eine umfassende Beurteilung des Schweregrads zu erreichen. Dieser erweiterte Beurteilungsrahmen wird bereits in den Instruktionen festgehalten (Tab. 1). Ferner wurden spezielle Maßnahmen und Beispielverhaltensweisen aus dem Voll- 
Tab. 1 Instruktionen des CGI-Corrections $(C G I-C)$

Der CGI-C ist ein klinisches Diagnostikinstrument, das auf dem CGI basiert und an die Besonderheiten des Vollzugs adaptiert wurde. Der allgemeine Schweregrad einer psychischen Störung wird auf einer Skala von 1 bis 7 anhand des Verlaufs der vergangenen 24h eingeschätzt

Der Begriff ,psychische Störung“ umfasst psychiatrische Erkrankungen (z. B. Depression/Schizophrenie), Missbrauch-/

Abhängigkeitserkrankungen, Persönlichkeitsstörungen, tiefgreifende Entwicklungsstörungen, Intelligenzminderungen, hirnorganische

Störungen, Zustände nach Gehirnverletzungen (z. B. Meningitis, Gehirnerschütterung) und vorübergehende Zustände wie z. B. Intoxikation oder

Entzugserscheinungen. Ebenso sind Verhaltensmanifestationen der genannten Störungen inbegriffen (wie z. B. Selbstverletzung, Selbstmordversuche, wiederholte oder ernsthafte Gewalttätigkeiten). Nicht beinhaltet sind klinische Auffälligkeiten, welche durch körperliche Erkrankungen verursacht werden, - außer es handelt sich um sog. Somatisierungen

Es ist zu beachten, dass etwaige Sicherheitsverfügungen einer Person nicht per se einen Einfluss auf die CGI-C-Einschätzung nehmen sollten. Der CGI-C sollte auf einem gesamtklinischen Eindruck basieren, mit allen in der jeweiligen Institution zur Verfügung stehenden Informationen. So sollte z. B. ein Gefangener, der sich in einer Hochsicherheitsabteilung befindet, unter Berücksichtigung der Möglichkeiten auf dieser Abteilung, und nicht im Vergleich mit den Möglichkeiten auf anderen Abteilungen oder denen der Gesellschaft eingeschätzt werden (d.h. Einschätzung im jeweiligen Referenzrahmen treffen). Wenn die Person jedoch aufgrund der psychischen Verfassung bzw. Symptomatik auf eine Krankenstation oder in eine Beobachtungszelle verlegt wurde, so hat dieses Einfluss auf die Beurteilung gemäß CGI-C

Für die Einschätzung sollten Informationen aus einer klinischen Einschätzung durch ein Interview (b) sowie fremdanamnestische Angaben (v. a. Justizvollzugsbeamte, Krankenpfleger*Innen, Akten bzw. Dokumentationen; a) herangezogen werden

In Situationen, in welchen Inhaftierte durch eine psychische Störung unfähig oder nicht gewillt sind, an einer klinischen Untersuchung teilzunehmen oder nur in einem eingeschränkten Maße teilnehmen können, muss die Beurteilung des CGI-C auf Informationen basieren, die sich durch die Beobachtung der Person in ihrem aktuellen Umfeld sowie fremdanamnestische Beobachtungen ergeben

zugssetting genommen, um die Alltagsfertigkeiten der Personen zu erfassen (z. B. ,häufig unter , besonderer Behandlung' (Sicherheitsverfügungen etc.) innerhalb der Abteilung/Institution“).

\section{Übersetzung des CGI-C}

Zur Erstellung einer validen Übersetzung orientierten sich die Autoren an den Richtlinien der WHO (https://www. who.int/substance_abuse/research_tools/translation/en/). In einem ersten Schritt wurde das Instrument durch die Autoren in die deutsche Sprache übersetzt. Während des Übersetzungsprozesses wurden bestimmte Items mit den Autoren des Originalinstruments diskutiert. Rücksprache- und Klärungsbedarf ergaben sich v.a. im Kontext der rechtlichen Unterschiede der kanadischen und deutschen Gesetzgebung sowie bei fehlenden Übersetzungsäquivalenten (z.B. „,collateral information“). Nach Klärung dieser Items erfolgte eine Rückübersetzung der ersten Version durch eine bilinguale Psychologin, die vorher nicht mit dem Instrument vertraut war. Diese Rückübersetzung wiederum wurde anschließend durch die Originalautoren evaluiert, um die inhaltliche Äquivalenz zur englischsprachigen Version überprüfen. Anhand dieser Kontrolle wurde die vorliegende Endversion der deutschsprachigen Version des CGI-C erstellt.

\section{Aufbau und Durchführung des CGI-C}

Der CGI-C soll eine Beurteilung des Schweregrads psychiatrischer Symptome von psychisch kranken Gefangenen ermöglichen. Anhand der Itembeschreibungen, die an Be- sonderheiten des Justizvollzugs angepasst und in fremdanamnestische und eigene Beobachtungen unterteilt wurden, können die Symptome/Beobachtungen/Einschätzungen auf einer Likert-Skala von 0 (,keine psychische Störung“) bis 7 (,schwerste psychische Störung“) eingeordnet werden. Als Beobachtungszeitraum empfiehlt es sich, die letzten 24h heranzuziehen (Tab. 2 zeigt als Beispiel die Kurzfassung des CGI-C). Für die Anwendung des CGI-C bedarf es keiner speziellen Schulung, jedoch muss der Anwendende über eine fundierte Kenntnis psychiatrischen, psychologischen und, zumindest basalen, juristischen Wissens verfügen und mit den Gegebenheiten des jeweiligen Vollzugs vertraut sein. Sollte dies nicht der Fall sein (z.B. externe Konsiliardienste, die nur wenige Berührungspunkte mit dem Vollzugssystem haben), wäre eine Unterstützung durch intramural tätige Personen (z.B. Abteilungsleitung, Bedienstete oder psychologischer Dienst) sinnvoll. Im Schnitt kann bei Vorliegen der relevanten Informationen ein Rating innerhalb von 5 min erfolgen. Die Version des deutschsprachigen CGI-C wird durch die Autoren gemäß der Creative Commons Attribution-NoDerivatives 4.0 International License (CC BY-ND 4.0) in der jeweils aktuellen Fassung zur Verfügung gestellt (Projektübersicht: https://www.researchgate.net/project/The-Clinical-GlobalImpression-Corrections-CGI-C-German). Die derzeitige Version findet sich zudem im elektronischen Anhang dieses Artikels.

\section{Datenerhebung}

Die Erhebung der Daten erfolgte an zwei Standorten: der Abteilung für Forensische Psychiatrie und Psychotherapie II an der LVR Klinik Köln und dem Institut für 
Tab. 2 Die Kurzfassung der deutschen Version des CGI-C (Billen et al. 2019)

\begin{tabular}{ll}
\hline Bewertung & Fremdanamnestische Angaben \\
\hline $\begin{array}{l}\text { 1 Keine } \\
\text { psychische }\end{array}$ & $\begin{array}{l}\text { Teilnahme an verfügbaren Angeboten/Arbeitsmöglichkeiten/Aufgaben, } \\
\text { gemessenes Sozialverhalten, kein Fehlverhalten oder kriminelle Aktivitäte }\end{array}$ \\
$\begin{array}{l}\text { 2 Grenz- } \\
\text { wertige } \\
\text { psychische }\end{array}$ & $\begin{array}{l}\text { Gutes Funktionsniveau. Probleme, die sich nicht auf die Alltagsfähigkeiten } \\
\text { auswirken und/oder vom Personal bemerkt werden }\end{array}$ \\
& \\
$\begin{array}{l}\text { 3 Leichte } \\
\text { psychische }\end{array}$ & $\begin{array}{l}\text { Gutes Funktionsniveau. Probleme, die das Funktionsniveau kaum beein- } \\
\text { trörung }\end{array}$
\end{tabular}

4 Mittelgradige psychische Störung

\section{Deutlich} (erkennbare) psychische Störung

6 Schwere psychische Störung

7 Schwerste psychische Störung
Teilweise dysfunktional. Könnte z. B. von den Justizvollzugsbeamten als „merkwürdig“ oder „unpassend/ungewöhnlich“ beschrieben werden, jedoch nicht explizit störend. Evtl. leichte Selbstverletzungen, laute Geräusche aus der Zelle (z. B. Schreien, Schlagen gegen Wände/Tür), ohne dabei physisch gewalttätig zu werden. Evtl. Schwierigkeiten mit der Hygiene. Funktionsniveau kann durch physikalische Manifestationen oder Entzugserscheinungen beeinträchtigt sein. Insgesamt aber im Kontakt kooperativ

Beeinträchtigtes Funktionsniveau, ggf. deutliche verbale Aggressionen, wiederholte Selbstverletzungen, ist unkooperativ im Umgang mit den Mitarbeitern/Mitarbeiterinnen, Schreien oder Randalieren in der Zelle für erhebliche Anteile des Tages. Angemessene Interaktionen mit anderen sind minimal. Häufig unter „,besonderer Behandlung“ (Sicherheitsverfügungen etc.) innerhalb der Abteilung/Institution, wenn die Person eine Gefahr für andere darstellt

Schwere Beeinträchtigungen des Funktionsniveaus. Justizvollzugsbeamte berichten über die Auffälligkeiten. Vermutlich treten Störungen des Tagesablaufes auf, hohe Wahrscheinlichkeit für oder tatsächlich stattfindende physische Gewalt, Verschmutzungen mit Exkrementen, Urinieren außerhalb der Toilette, aggressive Verhaltensweisen, die zu Zugriffen der Mitarbeiter*Innen führen - evtl. Absonderung. Impulsives und unvorhersehbares Verhalten. Häufig Sicherheitsverfügungen oder Absonderungen innerhalb der Abteilung/Institution, wenn die Person eine Gefahr für andere darstellt

Massive Beeinträchtigung der Funktionsfähigkeit. Eventuell physisch gewalttätig, Versuche potenziell tödlicher Selbstverletzungen oder keine Flüssigkeits-/Nahrungsaufnahme. Sehr impulsives und unvorhersehbares Verhalten. Sicherheitsverfügungen oder Absonderung innerhalb der Abteilung/Institution, wenn die Person eine Gefahr für andere darstellt
Beobachtung/Interview

Kein Hinweis auf psychische Störungen. Evtl. anamnestische Symptome oder Diagnosen, nicht gegenwärtig

Insgesamt gutes Funktionsniveau. (Evtl.) keine Vordiagnose/-behandlung, sehr leichte oder seltene Symptome, die nicht die Kriterien gemäß ICD-10 erfüllen oder die Person nur minimal belasten oder das Funktionsniveau minimal beeinträchtigen

Deutliche, aber leichte Symptome einer psychischen Erkrankung. Evtl. wird die Person bereits behandelt, weist jedoch noch (leichte) Restsymptomatik auf oder hat eine grenzwertige Intelligenz (z. B. Lernbehinderung)

Deutliche Symptome einer psychischen Erkrankung. Signifikantes Level an Belastung bzw. Leidensdruck, u. a. suizidale Gedanken, Selbstverletzungen und/oder psychotische Symptome können vorhanden sein. Ess- und Trinkverhalten sind adäquat. Vernachlässigen der persönliche Hygiene. Falls noch nicht stationär aufgenommen, ist dies vielleicht indiziert

Offensichtliche Symptome einer psychischen Erkrankung. Deutliche Beeinträchtigungen/ Belastung (Leidensdruck), mit möglichen Suizidgedanken, signifikante Selbstverletzungen und/oder psychotische Symptome. Essen und Trinken erfolgen ausreichend. Vernachlässigung der Hygiene. Ggf. stationäre Aufnahme notwendig, falls nicht bereits erfolgt

Schwere und dauerhafte Symptome einer psychischen Erkrankung. Person zeigt deutliche Einschränkungen, die das Funktionsniveau erheblich einschränken. Häufig zu schwer erkrankt, um an einem klinischen Interview teilzunehmen. Benötigt mit hoher Wahrscheinlichkeit eine stationäre Aufnahme, falls noch nicht erfolgt

Schwere und dauerhafte Symptome einer psychischen Erkrankung, evtl. katatone Zustände oder sehr starke Anspannung, Auftreten auffälliger Verhaltensweisen, wie z. B. potenziell tödliche Selbstverletzungen oder Essen von Exkrementen. Dringende Krankenhausaufnahme notwendig, wenn nicht bereits stationär untergebracht

Der Schweregrad einer psychischen Störung wird anhand des klinischen Eindrucks (z. B. Interview oder Beobachtung) und aller verfügbaren Informationen der jeweiligen Institution (z. B. durch Vollzugsbeamte) der letzten 24 h eingeschätzt. Der Begriff ,,psychische Störung“ umfasst psychiatrische Erkrankungen, Suchterkrankungen, Persönlichkeitsstörungen, tiefgreifende Entwicklungsstörungen, Intelligenzminderungen und deren Verhaltensmanifestationen (wie z. B. Selbstverletzungen oder Aggressivität)

Forensische Psychiatrie und Psychotherapie (ISFP) in Kiel. Die Einladung zur Studienteilnahme wurde an alle dort tätigen psychiatrisch-tätigen Ärzte und Psychologen ${ }^{1}$ gesendet. Jeder Rater erhielt eine digitale Version des CGI-C

\footnotetext{
1 Zur besseren Lesbarkeit wird im Text auf eine geschlechtsspezifische Differenzierung verzichtet, sofern sich die Ausführungen nicht explizit nur auf männliche, weibliche oder diverse Personen beziehen.
}

und 21 Fallvignetten. Die Fallvignetten wurden auf Basis realer Fälle von der Forschungsgruppe um Jones et al. (2019) entwickelt und anschließend überarbeitet, um alle Schweregrade der Symptome, verschiedene Diagnosen und Populationen abzudecken. Diese Fallvignetten wurden ebenfalls durch die Autoren übersetzt, um die Ermittlung der „Interrater“-Reliabilität möglichst analog zur Originalstudie von Jones et al. (2019) zu gestalten. Jede Fallvignette 
Tab. 3 Beurteilerübereinstimmung der Studienteilnehmer

\begin{tabular}{ll}
\hline Gwet's $\mathrm{AC}_{\mathrm{a}}$ & \\
\hline \% Übereinstimmung & 0,96 \\
$\%$ Erwartete Übereinstimmung & 0,74 \\
Koeffizent $^{\mathrm{a}}$ & 0,85 \\
Standardfehler & 0,02 \\
$95 \%$-KI & $0,81-0,90$ \\
Signifikanz & $0,00^{*}$ \\
\hline
\end{tabular}

Gwet's $A C^{2}$ Gwet's Agreement Coefficient

$* p<0,00$

aWerte über $0,81=$,exzellent“

wurde zusätzlich mit einem Kommentarfeld für mögliche Anmerkungen versehen. An weiteren Daten wurden lediglich die Berufsgruppe sowie der Standort der Rater erhoben; hierüber wurden die Teilnehmer/Teilnehmerinnen aufgeklärt und erklärten sich einverstanden. Insgesamt wurden 28 psychiatrische Ärzte/Ärztinnen und Psychologen/ Psychologinnen für die Erhebung kontaktiert.

\section{Ergebnisse}

Die Antwortrate von $71 \%$, welche sich auf 12 Psychologen und 8 Fachärzte für Psychiatrie und Psychotherapie verteilte, kann als sehr hoch eingeschätzt werden (Cunningham et al. 2015; Nulty 2008; Pickett et al. 2018). Die Berechnung der Beurteilerübereinstimmung durch Gwet's $\mathrm{AC}_{2}$ (Gwet 2014) ergab einen Wert von 0,82 (96\%-KI $0,74-0,91, p<0,001)$ über alle initialen Ratings der 21 Fallvignetten, welches eine sehr hohe Übereinstimmung darstellt (Tab. 3; Wongpakaran et al. 2013), vergleichbar mit den Ergebnissen der Originalstudie von Jones et al. (2019).

Die qualitativen Auswertungen der Fallvignetten und der zugehörigen Kommentare ergaben insgesamt 20 Fragen bzw. Anmerkungen, die sich in folgende Kategorien einteilen lassen: Fehlerkorrektur und Verständnisfragen (z. B. „Was ist ein Kalfaktor?“, „Das Wort ,nicht“ fehlt“), Unklarheiten bezüglich der vorliegenden psychischen Erkrankung (z.B. „Interesse ausreichend für Pädophilie-Diagnose?“, „Symptomatik ist unrealistisch“), Einordnungsschwierigkeiten (z. B. „,schwierige Entscheidung zwischen 4 und 5“, „chronifizierte Störung vs. hohes Funktionsniveau“) und Hinweise auf ggf. fehlende Operationalisierungen (z.B. „soziale Isolation ist nicht operationalisiert"). Die meisten Fragen stellten sich den Ratern im Hinblick auf die Fallvignetten, die z.T. im deutschen Gesetzeskontext ungültig waren (z.B. Begutachtung nach Verurteilung) oder unklar formuliert waren (z.B. welches Ausmaß der Flüssigkeitsaufnahme gegeben ist). Strukturelle Fragen oder Anmerkungen, die sich auf die Rating-Skala an sich bezogen, wurden nur 4-mal gestellt (d.h. fehlende Operationalisierung sozialer Isolation sowie Einordnungsschwierigkeiten).
Dies entspricht einer Frequenz von 0,01 \% (4 aus 420 möglichen Ratings).

\section{Diskussion}

Im intramuralen Arbeitsalltag mit psychisch kranken Gefangenen zeigen sich immer wieder Schwierigkeiten in der Beurteilung des Schweregrads psychischer Erkrankungen von Gefangenen, da die allgemeinpsychiatrischen Skalen nicht auf die Besonderheiten des Justizvollzuges angepasst sind. Aber auch vor dem Hintergrund des Äquivalenzprinzips, welches eine Gesundheitsversorgung von Gefangen erfordert, die in Qualität und Umfang der Versorgung außerhalb des Vollzugs entspricht (Charles und Draper 2011; Jotterand und Wangmo 2014; Lines 2006; Melis 2006; Niveau 2007; Till et al. 2014; Wilson 2004), ist es notwendig, Instrumente zu erschaffen, die psychiatrische Erkrankungen erfassen und deren Schweregrad zuverlässig beurteilen können. Nur auf diese Weise können qualitative und spezifisch zugeschnittene Behandlungsmaßnahmen angeboten und evaluiert werden. Um diesen Defiziten zu begegnen und die Entwicklung geeigneter Behandlungsmaßnahmen zu unterstützen, wurde der im angelsächsischen Raum entwickelte CGI-C ins Deutsche übersetzt.

In einem ersten Schritt galt es nun zu überprüfen, ob sich die positiven Ergebnisse der kanadischen Autoren (Jones et al. 2019) im deutschsprachigen Raum replizieren lassen. So wies die Studie von Jones et al. (2019) eine sehr hohe Beurteilerübereinstimmung in Bezug auf die verwendeten Fallvignetten auf (Gwet's $A C_{2}=0,89,95 \%-K I$ 0,84-0,92, $p<0,001$, ,second rating "), welche sich nur geringfügig von der hiesigen Studie unterscheidet (Gwet's $A C_{2}=0,82$ ): Beide Werte stellen eine sehr hohe Übereinstimmung dar. Im Gegensatz zu der Studie von Jones et al. (2019) erfolgte bisher jedoch keine Überprüfung der Test-Retest-Reliabilität und der Beurteilerübereinstimmung anhand von realen Patienten.

Dennoch können die ersten Ergebnisse dieser Studie zu Reliabilität und Validität des CGI-C als vielversprechend betrachtet werden: Die Beurteilerübereinstimmung ist durchweg hoch, ohne dass zuvor eine spezifische Schulung der Teilnehmer zum CGI-C erfolgen musste. Es sollte jedoch beachtet werden, dass es sich bei den Ratern um ausgebildete Ärzte und Psychologen handelt, deren Erfahrung im forensischen Kontext im Mittel bei 6,4 Jahren lag (Min.:1, Max.: 20). Außerdem wurden konstruierte Fallvignetten verwendet, sodass eine Überprüfung der Gütekriterien anhand realer Fälle noch erfolgen muss.

Das Instrument selbst wurde als schnell (zwischen einer und 10 min Ratingzeit) und einfach durchführbar beschrieben, welches sich auch als Ergebnis der Studie von Jones et al. (2019) wiederfindet. Somit erscheint auch die deut- 
sche Version des CGI-C als ein effizientes Instrument zur Erfassung der Schwere einer psychiatrischen Störung im forensischen Setting (v. a. Justizvollzugsanstalten) geeignet. Die einzelnen Items sind - in Bezug auf die Fallvignetten - ausreichend operationalisiert, sodass sie nur vereinzelt Nachfragen der Rater hervorriefen. Die meisten Rückfragen ergaben sich zu den Fallvignetten, die von den Autoren aus dem Englischen übersetzt wurden. Diese wurden z.T. aufgrund der Symptomatik oder im Kontext der deutschen Gesetzgebung als unrealistisch bewertet, wirkten sich jedoch nicht auf die getroffenen Einschätzungen aus, sodass diese seitens der Autoren als Verständnisfragen gewertet wurden.

\section{Klinische Implikationen und Einsatzgebiete}

Das Rational des vorliegenden Instruments und der darauf aufbauenden Studie ist eine einfache, schnelle und valide Einschätzung des Schweregrads psychiatrischer Symptome inhaftierter Personen. Anhand dieser Einschätzung soll es erleichtert werden, die Indikationsstellung für intramurale Behandlungsangebote rational und zuverlässig stellen zu können. Zudem wäre es denkbar, dass eine vorläufige Einschätzung auch durch nicht explizit medizinisch-psychologisch geschultes Personal (z. B. Sozialdienst) durchgeführt werden kann, um abhängig von der Auswertung eine Behandlung oder Vorstellung bei einem (Fach-)Arzt in die Wege leiten zu können.

\section{Limitationen und Ausblick}

Der Fokus liegt in dieser Studie auf der ersten Übersetzung des Originalinstruments sowie der Validierung anhand von Fallvignetten - eine Übertragung in den klinischen Alltag des Vollzugs muss somit noch erfolgen. Die Überprüfung im Rahmen der vorliegenden Studie erfolgte außerdem ausschließlich durch Psychologinnen und Ärzte; dies könnte die Übertragbarkeit auf andere Berufsgruppen des Vollzugs limitieren. Somit sollten durch weitere Studien die Validität und Reliabilität des CGI-C mit anderen Professionen (Pflegekräfte und Beamte des Vollzugsdienstes) überprüft und bestätigt werden. Ferner handelte es sich bisher um Experten-Ratings auf Basis konstruierter Fallbeispiele, deren Übertragbarkeit auf reale Populationen nur bedingt vorliegt. Gleichwohl zeigten die ersten Studienergebnisse der kanadischen Version anhand realer Fälle, dass das Instrument valide eingesetzt werden konnte (Jones et al. 2019). Dies lässt den Rückschluss zu, dass auch die übersetzte Version entsprechend verwendet werden kann. Dennoch sollte dies in weiteren Studien überprüft werden. In gleicher Weise steht auch noch der Vergleich mit ähnlichen Instrumen- ten zur Erhebung der Validität aus, so wäre zum Beispiel ein Vergleich der Einschätzungen anhand des CGI-C mit den Ergebnissen der Symptom-Checkliste von Derogatis (SCL-90R, Franke 2002) oder der Core Outcome Measures (CORE-OM, Evans et al. 2000) denkbar.

Für die weitere Validierung des Instruments ist geplant, diesen Einschränkungen mithilfe weiterer Untersuchungen zu beheben. So werden bereits Vorbereitungen getroffen, das Instrument auf einer intramuralen psychiatrischen Station des Landes Schleswig-Holsteins einzusetzen und somit zur weiteren Validierung beizutragen. Hier ist es ein Anliegen der Autoren, die Forschung auf reale Fälle zu übertragen, um die Validität und Reliabilität des Instruments im Alltag zu überprüfen und die Intensität der Symptome bzw. Qualität der Funktionseinschränkungen zu erfassen. Zudem sind weitere Studien zur Anwendbarkeit im forensischen Arbeitsalltag sowie evtl. Follow-ups der beurteilten Personen geplant, die zu einer psychologischen und/oder psychiatrischen Behandlung überwiesen wurden.

Da das Ausgangsinstrument nicht nur diagnose-, sondern auch altersgruppenübergreifend angewendet werden kann, sollten die Reliabilität und Validität des CGI-C auch in den verschiedenen forensischen Settings (z. B. Justizvollzugsanstalten für Jugendliche und/oder Frauen) überprüft werden.

Eine grundsätzliche Limitation des vorliegenden Instruments könnte darin gesehen werden, dass es keine umfassenden diagnostischen Untersuchungen ersetzen kann. Diesem ist jedoch entgegenzusetzen, dass der CGI-C gerade dafür konzipiert wurde, eine einfache und schnelle Einschätzung des Schweregrads psychiatrischer Symptome zu ermöglichen, um eine schnelle, aber dennoch empirisch validierte Differenzierung treffen zu können, für welchen Patienten eine solche umfassende Untersuchung vorgesehen werden sollte.

Um die vollständige Validierung des Instruments zu gewährleisten, laden die Autoren deshalb dazu ein, das Instrument bereits jetzt zu verwenden (https://www.researchgate. net/project/The-Clinical-Global-Impression-CorrectionsCGI-C-German) und eigene Forschungsarbeiten unter Rücksprache durchzuführen. Sofern möglich, bitten wir in diesem Rahmen darum, die Daten mit den Autoren für weitere Erhebungen der Gütekriterien anonymisiert zu teilen. Somit soll es ermöglicht werden, deutschlandweit neben den bereits bekannten hohen Prävalenzen von psychischen Belastungen in Gefängnissen auch ein differenzierteres Bild bezüglich der damit einhergehenden Funktionseinbußen der Inhaftierten aufzeigen zu können. Auf dieser Basis können dann bestehende Behandlungsmaßnahmen angepasst sowie neue entwickelt werden, um der Erfüllung des Äquivalenzprinzips in der psychiatrischen Versorgung in Gefängnissen ein Stück näherzukommen. 
Danksagung Die Autoren bedanken sich bei dem Chefarzt der Abteilung für Forensische Psychiatrie und Psychotherapie II der LVR-Klinik Köln, Herrn Dr. Prüter-Schwarte, und dem Direktor der Abteilung Forensische Psychiatrie und Psychotherapie des Instituts für Sexualmedizin und Forensische Psychiatrie und Psychotherapie (ISFP) in Kiel, Herrn Prof. Dr. Huchzermeier, für die Zusammenarbeit.

Funding Open Access funding provided by Projekt DEAL.

\section{Einhaltung ethischer Richtlinien}

Interessenkonflikt C. Billen, M.A. Schulte-Ostermann und C. Huchzermeier geben an, dass kein Interessenkonflikt besteht.

Ethische Standards Für diesen Beitrag wurden von den Autoren keine Studien an Menschen oder Tieren durchgeführt. Für die aufgeführten Studien gelten die jeweils dort angegebenen ethischen Richtlinien. Für Bildmaterial oder anderweitige Angaben innerhalb des Manuskripts, über die Patienten zu identifizieren sind, liegt von ihnen und/oder ihren gesetzlichen Vertretern eine schriftliche Einwilligung vor.

Open Access Dieser Artikel wird unter der Creative Commons Namensnennung 4.0 International Lizenz veröffentlicht, welche die Nutzung, Vervielfältigung, Bearbeitung, Verbreitung und Wiedergabe in jeglichem Medium und Format erlaubt, sofern Sie den/die ursprünglichen Autor(en) und die Quelle ordnungsgemäß nennen, einen Link zur Creative Commons Lizenz beifügen und angeben, ob Änderungen vorgenommen wurden.

Die in diesem Artikel enthaltenen Bilder und sonstiges Drittmaterial unterliegen ebenfalls der genannten Creative Commons Lizenz, sofern sich aus der Abbildungslegende nichts anderes ergibt. Sofern das betreffende Material nicht unter der genannten Creative Commons Lizenz steht und die betreffende Handlung nicht nach gesetzlichen Vorschriften erlaubt ist, ist für die oben aufgeführten Weiterverwendungen des Materials die Einwilligung des jeweiligen Rechteinhabers einzuholen.

Weitere Details zur Lizenz entnehmen Sie bitte der Lizenzinformation auf http://creativecommons.org/licenses/by/4.0/deed.de.

\section{Literatur}

Billen C, Schulte Ostermann MA, Huchzermeier C (2019) The Clinical Global Impression - Corrections (CGI-C) German Version, Kurzfassung. Technic Rep. https://doi.org/10.13140/RG.2.2.20593. 84320

Charles A, Draper H (2011) Equivalence of care'in prison medicine: is equivalence of process the right measure of equity? J Med Ethics. https://doi.org/10.1136/medethics-2011-100083

Cunningham CT, Quan H, Hemmelgarn B, Noseworthy T, Beck CA, Dixon E, Jetté N et al (2015) Exploring physician specialist response rates to web-based surveys. BMC Med Res Methodol 15(1):32

Evans C, Mellor-Clark J, Margison F, Barkham M, Audin K, Connell J, Mc-Grath G (2000) CORE: clinical outcomes in routine evaluation. J Mental Health 9(3):247-255

Fazel S, Danesh J (2002) Serious mental disorders in 23000 prisoners: a systematic review of 62 surveys. Lancet 359:545-548

Fazel S, Seewald K (2012) Severe mental illness in 33588 prisoners worldwide: systematic review and meta-regression analysis. $\mathrm{Br}$ J Psychiatry 200(5):364-373. https://doi.org/10.1192/bjp.bp.111. 096370

Fazel S, Bains P, Doll H (2006) Substance abuse and dependence in prisoners: a systematic review. Addiction 101(2):181-191. https:// doi.org/10.1111/j.1360-0443.2006.01316.x
Fazel S, Hayes A, Bartellas K, Clerici M, Trestman R (2016) Mental health of prisoners: prevalence, adverse outcomes, and interventions. Lancet Psychiatry 3(9):871-881. https://doi.org/10.1016/ S2215-0366(16)30142-0

Franke GH (2002) Die Symptom-Checkliste von Derogatis (SCL-90R) - Deutsche Version - Manual. Weinheim: Beltz Test

Goff A, Rose E, Rose S, Purves D (2007) Does PTSD occur in sentenced prison populations? A systematic literature review. Criminal Behav Ment Health 17(3):152-162. https://doi.org/10.1002/ cbm. 653

Gwet KL (2014) Handbook of inter-rater reliability: The definitive guide to measuring the extent of agreement among raters. Advanced Analytics, LLC

Huchzermeier C, Schulte-Ostermann MA, Folgmann S (2016) Neues aus der Anstalt: Die „psychiatrische Sprechstunde“ in der JVA Neumünster - Minimalversorgung psychischer Störungen. RPsych 2(2):190-205

Janssen B, Janner M, Schneider F, Gaebel W, Burgmann C, Held T, Mecklenburg H et al (1998) Qualitatsindikatoren der stationären Behandlung schizophrener Patienten. Psychiat Prax 25(6):303309

Jones RM, Patel K, Moscovici M, McMaster R, Glancy G, Simpson AIF (2019) Adaptation of the clinical global impression for use in correctional settings: the CGI-C. Front Psychiatry 10:687. https://doi.org/10.3389/fpsyt.2019.00687

Jotterand F, Wangmo T (2014) The principle of equivalence reconsidered: assessing the relevance of the principle of equivalence in prison medicine. Am J Bioeth 14(7):4-12

Kamath J, Zhang W, Kesten K, Wakai S, Shelton D, Trestman R (2013) Algorithm-driven pharmacological management of bipolar disorder in Connecticut prisons. Int J Offender Ther Comp Criminol 57(2):251-264

Köhler D, Heinzen H, Hinrichs G, Huchzermeier C (2009) The prevalence of mental disorders in a German sample of male incarcerated juvenile offenders. Int $\mathrm{J}$ Offender Ther Comp Criminol 53(2):211-227

Lines R (2006) From equivalence of standards to equivalence of objectives: the entitlement of prisoners to health care standards higher than those outside prisons. Intl Jnl of Prisoner Health 2(4):269-280

MacKain SJ, Baucom T (2008) Medication management skills for mentally ill inmates: training is not enough. J Behav Anal Offender Vict Treat Prev 1(1):118

Melis M (2006) From the principle of equivalence to the practice of care: thoughts from the 9th ENDIPP conference. Intl Jnl of Prisoner Health 2(4):303-305

Nelson E-L, Zaylor C, Cook D (2004) A comparison of psychiatrist evaluation and patient symptom report in a jail telepsychiatry clinic. Telemed J E Health 10(Supplement 2):54-59

Niveau G (2007) Relevance and limits of the principle of "equivalence of care" in prison medicine. J Med Ethics 33(10):610-613

Nulty DD (2008) The adequacy of response rates to online and paper surveys: what can be done? Assess Eval High Educ 33(3):301-314

Pickett J, Cullen F, Bushway SD, Chiricos T, Alpert G (2018) The response rate test: Nonresponse bias and the future of survey research in criminology and criminal justice. SSRN 3103018

Rittmannsberger H, Sulzbacher A, Foff C, Zaunmüller T (2014) Heavy User stationärer psychiatrischer Behandlung: Vergleich nach Diagnosegruppen. Neuropsychiatrie 28(4):169-177

Scalarum Collegium Internationale Psychiatriae (2015) Internationale Skalen für Psychiatrie, 6. Aufl. Hogrefe, Göttingen

von Schönfeld CE, Schneider F, Schröder T, Widmann B, Botthof U, Driessen M (2006) Prävalenz psychischer Störungen, Psychopathologie und Behandlungsbedarf bei weiblichen und männlichen Gefangenen. Nervenarzt 77(7):830-841. https://doi.org/10.1007/ s00115-005-1946-1 
Schulte-Ostermann MA, Huchzermeier C (2019) Gefängnispsychiatrie zwischen Bedarf und Wirklichkeit: Die psychiatrische Tagesklinik in der JVA Neumünster. RPsych 5(1):42-60

Till A, Forrester A, Exworthy T (2014) The development of equivalence as a mechanism to improve prison healthcare. J R Soc Med 107(5):179-182

Trestman RL, Ford J, Zhang W, Wiesbrock V (2007) Current and lifetime psychiatric illness among inmates not identified as acutely mentally ill at intake in Connecticut's jails. J Am Acad Psychiatry Law Online 35(4):490-500
Wilson S (2004) The principle of equivalence and the future of mental health care in prisons. Br J Psychiatry 184(1):5-7

Wongpakaran N, Wongpakaran T, Wedding D, Gwet KL (2013) A comparison of Cohen's Kappa and Gwet's AC1 when calculating inter-rater reliability coefficients: a study conducted with personality disorder samples. BMC Med Res Methodol. https://doi.org/ 10.1186/1471-2288-13-61 